\title{
Manejo quirúrgico de la hidatidosis hepática en esta última década: ¿Qué hemos aprendido?
}

\author{
Pedro Pablo Pinto G.
}

Servicio de Cirugía Hospita Regional de Coyhaique. Región de Patagonia, Chile.

Recibido 2021-01-15y aceptado 2021-02-22

Correspondencia a: Dr. Pedro Pablo Pinto G. pedropablopatricio@gmail.

\section{Surgical management of liver hydatidosis in the last decade: What have we learned?}

The clinical management of hepatic hydatidosis is complex and is fundamentally based on three pillars: surgical, percutaneous and pharmacological; which can sometimes be complementary. Therapy should be defined based on the characteristics, location and type of cyst; to which must be added the experience of the medical team, available resources and the adherence of patients to long-term monitoring. Surgery is the most used treatment, percutaneous techniques can possibly replace surgery in specific cases. However, despite advances in surgical techniques, in the use of chemotherapy and other treatments, the management of liver hydatidosis continues to be a major problem; mainly due to the lack of consensus on which procedure to use is better. The objective of this presentation is to present the wide range of existing therapies in the current management of hepatic hydatidosis and some suggestions in the use of each one of them. Key words: cystic echinococcosis; ultrasonographic classification; surgery; percutaneous treatment; laparoscopy; watch and wait.

\section{Resumen}

El manejo clínico de la hidatidosis hepática es complejo y se basa fundamentalmente en tres pilares: quirúrgico, percutáneo y farmacológico; los cuales en ocasiones pueden ser complementarios. La terapia debe definirse en función de las características, ubicación y tipo de quiste; a lo cual debe sumarse la experiencia del equipo médico, recursos disponibles y la adherencia de los pacientes al monitoreo a largo plazo. La cirugía es el tratamiento más utilizado, las técnicas percutáneas posiblemente puedan reemplazar a la cirugía en casos específicos. Sin embargo, a pesar de los avances en las técnicas quirúrgicas, en el uso de la quimioterapia y otros tratamientos, el manejo de hidatidosis hepática sigue siendo un problema importante, debido principalmente a la falta de consenso sobre qué procedimiento a utilizar es mejor. El objetivo de esta presentación es dar a conocer el amplio abanico de terapias existentes en el actual manejo de la hidatidosis hepática, así como indicar algunas sugerencias en la utilización de cada una de ellas.

Palabras clave: echinococcosis quística; cirugía; tratamiento percutáneo; laparoscopía; observar y esperar.

\section{Introducción}

La enfermedad hidatídica es una zoonosis causada por la tenia Echinococcus granulosus senso lato; los perros son los huéspedes definitivos y los humanos un huésped intermediario accidental ${ }^{1}$. Debido a su impacto en términos de morbilidad, prevalencia en áreas en desarrollo y la falta de inversión en investigación; la equinococosis está incluida en la lista de enfermedades tropicales desatendidas de la Organización Mundial de la Salud (OMS) ${ }^{2}$.
Aunque la echinococcosis quística (EQ) tiene una distribución mundial, la mayoría de los casos humanos se concentran en América del Sur, Europa (países mediterráneos), África del Norte y del Este, Oriente Medio, Países de Asia Central y Occiden$\mathrm{tal}^{3}$.

En los seres humanos, puede dar lugar a un amplio espectro de manifestaciones clínicas, que van desde infecciones asintomáticas hasta la enfermedad fatal. El manejo de la EQ es complejo, no existiendo actualmente consenso, a pesar de las recomendaciones dadas por la $\mathrm{OMS}^{4}$. 
Esencialmente hay cuatro categorías de tratamientos, que a menudo se utilizan combinadamente: cirugía abierta, mínimamente invasiva, terapia médica y "observar y esperar" $(W \& W)$. El manejo va a variar de acuerdo con las características del paciente, del quiste y de los recursos disponibles ${ }^{4}$.

\section{¿Cuál es la utilidad de las imágenes en el manejo quirúrgico de echinococcosis hepática?}

La ultrasonografía (US) sigue siendo la piedra angular del diagnóstico, la estadificación y el seguimiento de la enfermedad hidatídica. A través de la US se ha logrado un progreso sustancial en la comprensión de la evolución natural y el tratamiento de los quistes hidatídicos hepáticos $(\mathrm{QHH})$. Esto, hasta el punto de que la decisión del tratamiento puede basarse cada vez más en las etapas del quiste.

En 2003, la OMS publicó una clasificación estandarizada de las imágenes ecográficas del $\mathrm{QHH}^{5}$, fácil de usar, no invasiva, sensible y específica. La clasificación de la OMS consta de seis estadios. Los quistes CE1, CE2 y CE3 muestran signos patognomónicos, mientras que los quistes CL, CE4, CE5 son lesiones quísticas tempranas indiferenciadas, caseadas y calcificadas respectivamente; las que presentan características sugerentes, pero no patognomónicas. En general, CE1, CE2 se consideran "activos", C3 como "transitorios" y CE4 y CE5 como "inactivos".
Dado que las decisiones de tratamiento se basan en las imágenes, es importante saber cómo se traduce esta clasificación US cuando se solicita, ya sea una tomografía axial computada (TC) y/o una resonancia magnética (RM). Stojkovic y cols. ${ }^{6}$, demostraron que la RM tiene un muy buen nivel de concordancia en los estadios CE1 a CE4, pero no así en el CE5, donde presenta deficiencia en la identificación de los detalles de la pared del quiste. La TC, sin embargo, es superior a la US y a la RM en los quistes CE5, pero inferior a la RM y al US en las otras etapas quísticas (Figura 1).

Por lo tanto, cuando no se puede realizar una US debido a la ubicación del quiste, o por razones específicas del paciente, es preferible la $\mathrm{RM}$ a la $\mathrm{TC}^{6}$.

\section{¿Cuáles son las terapias actuales del quiste hidatídico hepático?}

Hay cuatro opciones: cirugía abierta, cirugía mínimamente invasiva, observación y espera (W\&W) y terapia médica (Figura 2).

\section{Cirugía}

La cirugía debe adaptarse al paciente, a la anatomía, experiencia del cirujano y a las instalaciones hospitalarias. No está claro qué opción de tratamiento es la más segura y eficaz.

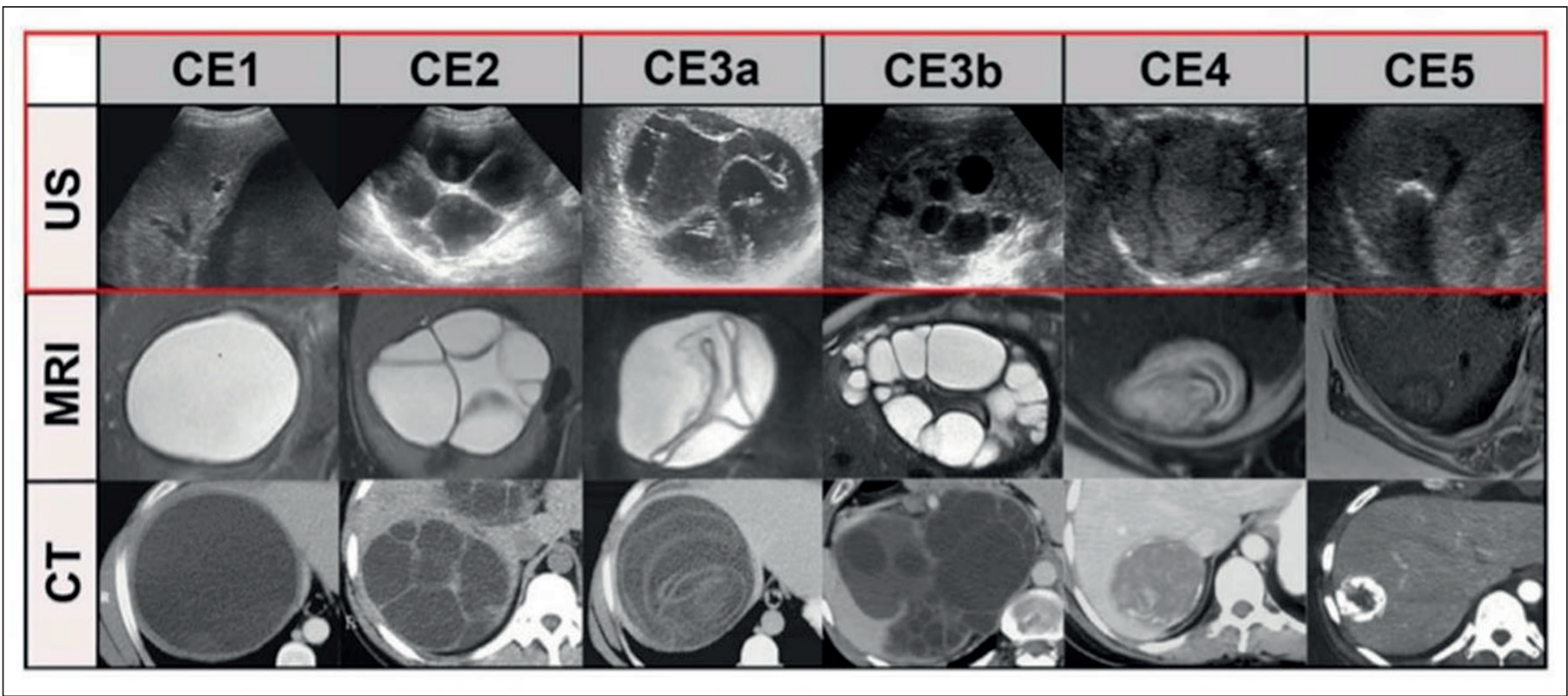

Figura 1. US: ultrasonografía; MRI: resonancia magnética; CT: tomografía computada. PLOS Neglected Tropical Diseases / www.plosntds.org. October 2012;6(10):e1880 


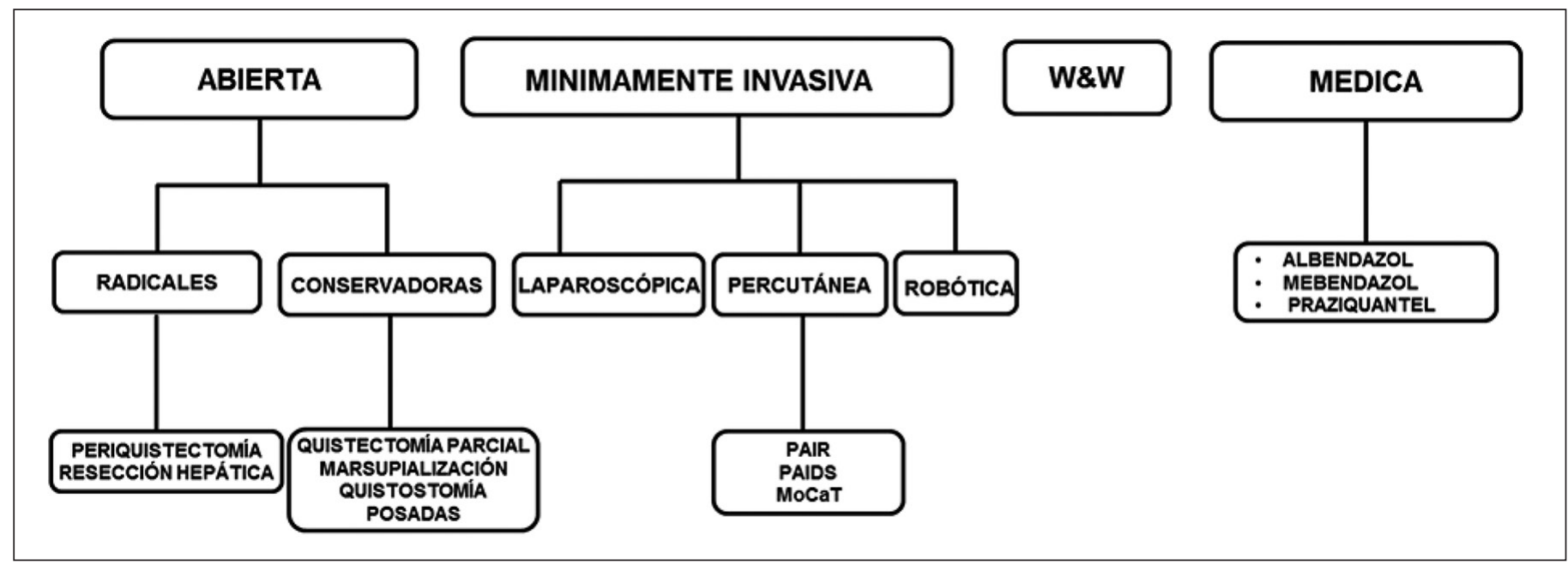

Figura 2. W\&W: Observación y espera.

Las técnicas clásicas, entendidas como las no radicales o conservadoras, han facilitado el manejo de la EQ en hospitales que no cuentan con unidades de cirugía hepatobiliar, por ser técnicas menos complejas, que habitualmente requieren menos tiempo quirúrgico y son más seguras. Sin embargo, las tasas de recurrencia (4\%-25\%) y complicaciones posoperatorias $(6 \%-47 \%)$ tienden a ser más altas que las técnicas radicales? .

La cirugía radical se refiere a la extirpación del quiste junto con la membrana periquística e incluso puede incluir la resección hepática ${ }^{7}$. Estos procedimientos deben ser realizados por cirujanos con experiencia en centros de cirugía hepatobiliar. La cirugía radical tiene la ventaja de tener menos complicaciones posoperatorias $(0 \%-26 \%)$ y bajas tasas de recurrencia $(0 \%-3 \%)$ pero alto riesgo intraoperatorio ${ }^{8}$.

De hecho, los tratamientos quirúrgicos se pueden resumir como un debate entre dos enfoques opuestos:

1) Conservador: apoyado por cirujanos generales de áreas endémicas, que enfatizan la necesidad de un tratamiento de primera línea seguro y confiable.

2) Radical: apoyado principalmente por cirujanos hepatobiliares que enfatizan la importancia de la completa extirpación de la periquística.

Los procedimientos conservadores son más seguros y más fáciles de realizar. Incluyen la extracción del contenido del quiste, la eliminación de la cúpula (con/sin omentoplastia, capitonaje, marsupialización, drenaje u otros métodos para el manejo de la cavidad residual) ${ }^{9}$. Entre todos los procedimientos conservadores, la quistectomía parcial es la mejor opción, considerándose fácil y segura; pero deja una cavidad residual que expone a complicaciones específicas (Figura 3).

La cirugía radical se refiere a la extirpación del quiste junto a la membrana periquística (periquistectomía) y también puede incluir la resección del hígado si está indicado. Algunos cirujanos sugieren que, siempre que sea posible, los métodos radicales deberían ser el pilar del tratamiento quirúrgico. Afirman que las ventajas son tasas más bajas de complicaciones tempranas y tardías; sin embargo, no existe una clara evidencia de esta afirmación ${ }^{10}$ (Figura 4).

A su vez, la resección hepática puede ser considerada cuando están comprometidas las principales estructuras biliares, cuando el parénquima hepático ha sido reemplazado por el quiste, en quistes múltiples localizados en un lóbulo, quistes pequeños localizados periféricamente o bien ante la presencia de un quiste pediculado ${ }^{11}$ (Figura 5).

\section{Recomendaciones}

- Siempre que sea posible, las técnicas radicales son preferibles a las técnicas conservadoras.

- El abordaje debe ser simple, evitando la filtración y protegiendo la cavidad peritoneal.

\section{Tratamiento laparoscópico}

Los peligros más importantes en el abordaje laparoscópico son el limitado espacio para la instrumentación, la complejidad en el control del derrame después de la punción del quiste, la dificultad en la 
aspiración de contenidos quísticos espesos, la posibilidad del aumento del riesgo de contaminación del líquido hidatídico por el uso del neumoperitoneo, la dificultad del control de la hemorragia y finalmente la pronunciada curva de aprendizaje $\mathrm{e}^{12,13}$.

Los estrictos criterios de selección, basados en la ubicación y el tamaño de la lesión, la experiencia en cirugía mínimamente invasiva y la instrumentación disponible, son extremadamente importantes para considerar un abordaje laparoscópico de la hidatidosis.

En centros con amplia experiencia en el tratamiento de la hidatidosis hepática, los criterios de exclusión son la localización intraparenquimatosa, quistes en la proximidad de vasos principales, quistes recidivados y una pared quística calcificada y gruesa $^{13,14}$.

El número, tamaño y ubicación de los quistes no se consideran contraindicaciones absolutas, aunque se acepta que el abordaje laparoscópico es más complejo cuando los quistes están en los segmentos centrales posteriores y cuando hay quistes grandes y múltiples ${ }^{15}$. El aumento de la habilidad en la cirugía hepática laparoscópica ha llevado a reevaluar las contraindicaciones inicialmente postuladas. En la actualidad no existen segmentos que se consideren inaccesibles, aunque la localización posterior y las hepatectomías mayores son técnicamente más exigentes y tienen tasas de conversión más elevadas ${ }^{16-18}$ (Figura 6).

El primer caso de abordaje laparoscópico para el tratamiento del QHH se describió en 1992 por Katkhouda y cols. ${ }^{19}$, pero debido al riesgo potencial de derrame y anafilaxia durante el procedimiento, la difusión de esta técnica fue inicialmente limitada. Desde entonces, se han publicado varios estudios que han reportado buenos resultados; sin embargo, la mayoría de estos estudios se basan en procedimientos laparoscópicos conservadores ${ }^{20}$ y son escasas las publicaciones que incluyen procedimientos radicales $^{21}$.

Tuxum y cols. ${ }^{22}$, revisaron la literatura mundial sobre el tratamiento laparoscópico de la EQ. El estudio incluyó a 914 pacientes con 1.116 quistes. El rango de edad fue de 3 a 70 años, el rango de diámetro del quiste fue de 3-18 cm (el 84\% tenía entre $5-10 \mathrm{~cm})$. Los procedimientos realizados fueron mayoritariamente conservadores (75\%): Quistectomía (60\%), periquistectomía parcial (15\%) y solo en un $10,8 \%$ se realizaron técnicas radicales: periquistectomía (8\%), lobectomía izquierda (1\%), segmentectomía izquierda (1\%). La tasa de conversión fue de 4,92\%. El tiempo operatorio osciló entre 50 y 144 min, el cual disminuyó a medida que

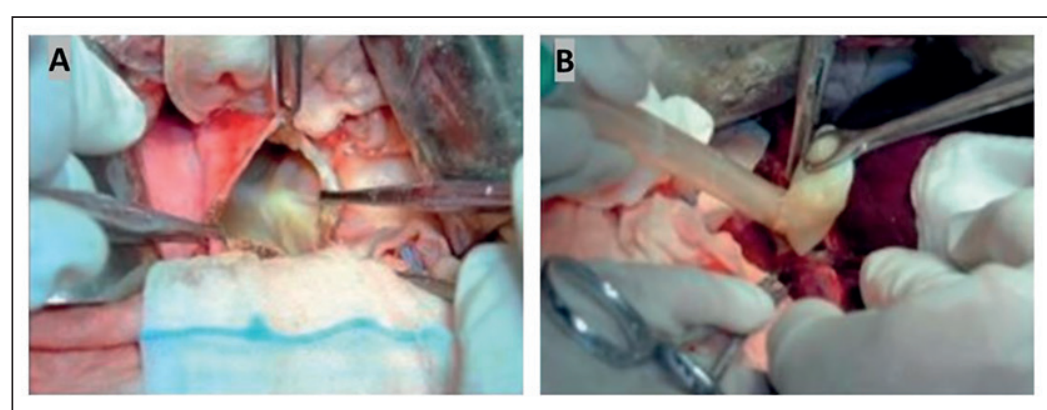

Figura 3. Quistectomía parcial. A: QHH abierto; B: Extracción de la membrana parasitaria.
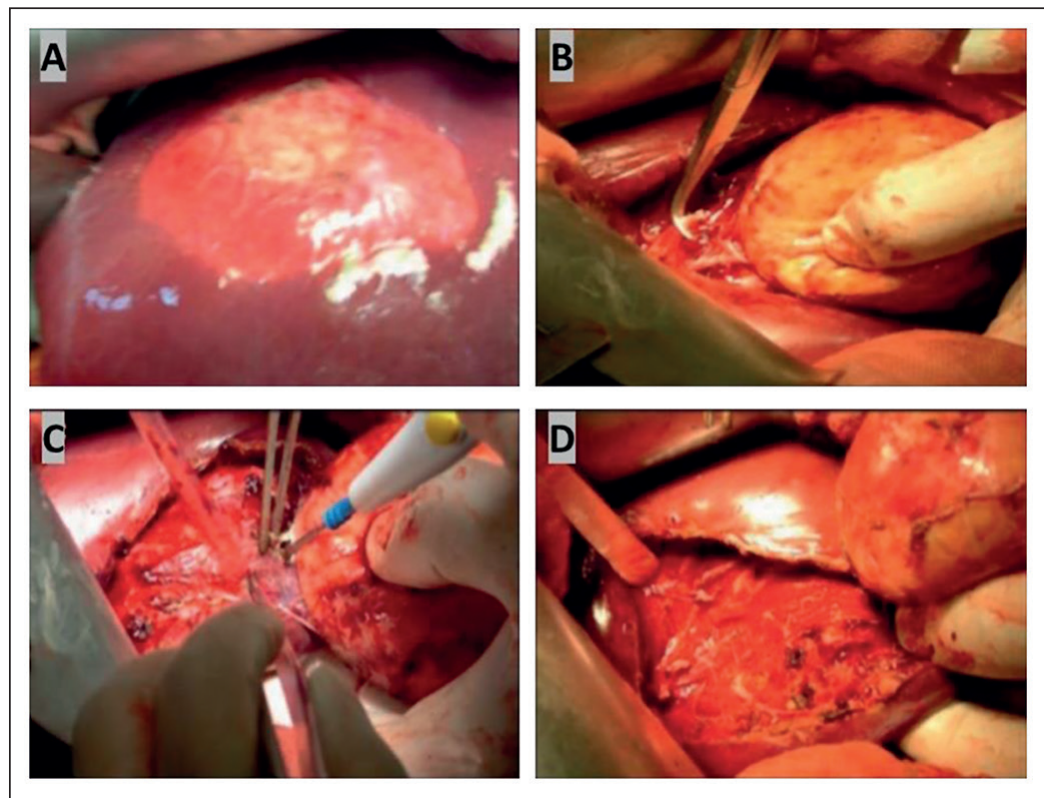

Figura 4. Periquistectomía total. A: QHH, S-VIII; B: Despegue periquística del parénquima hepático; C: Ligadura de los vasos vasculares y biliares; D: Extirpación completa del QHH.

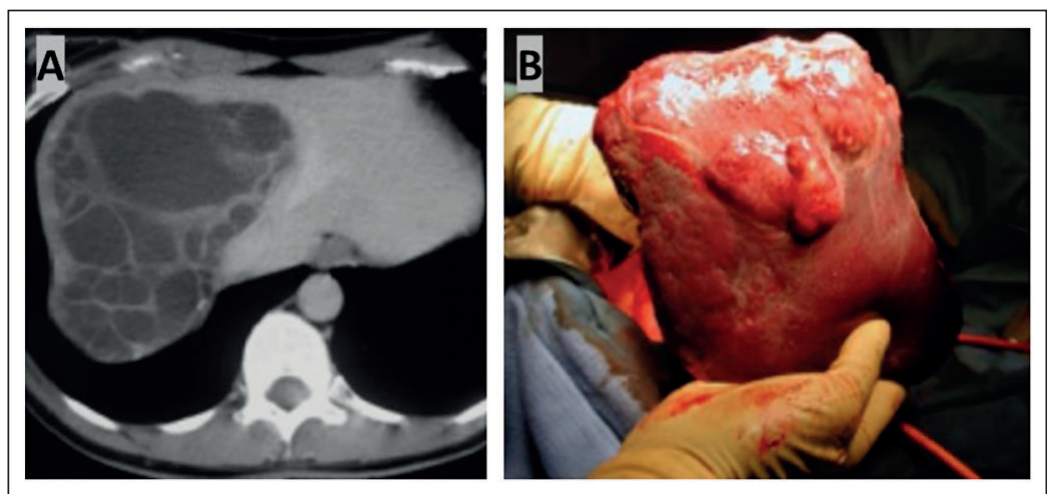

Figura 5. Hepatectomía. A: Imagen QHH LHD; B: Pieza operatoria. 


\section{CIRUGíA AL díA}
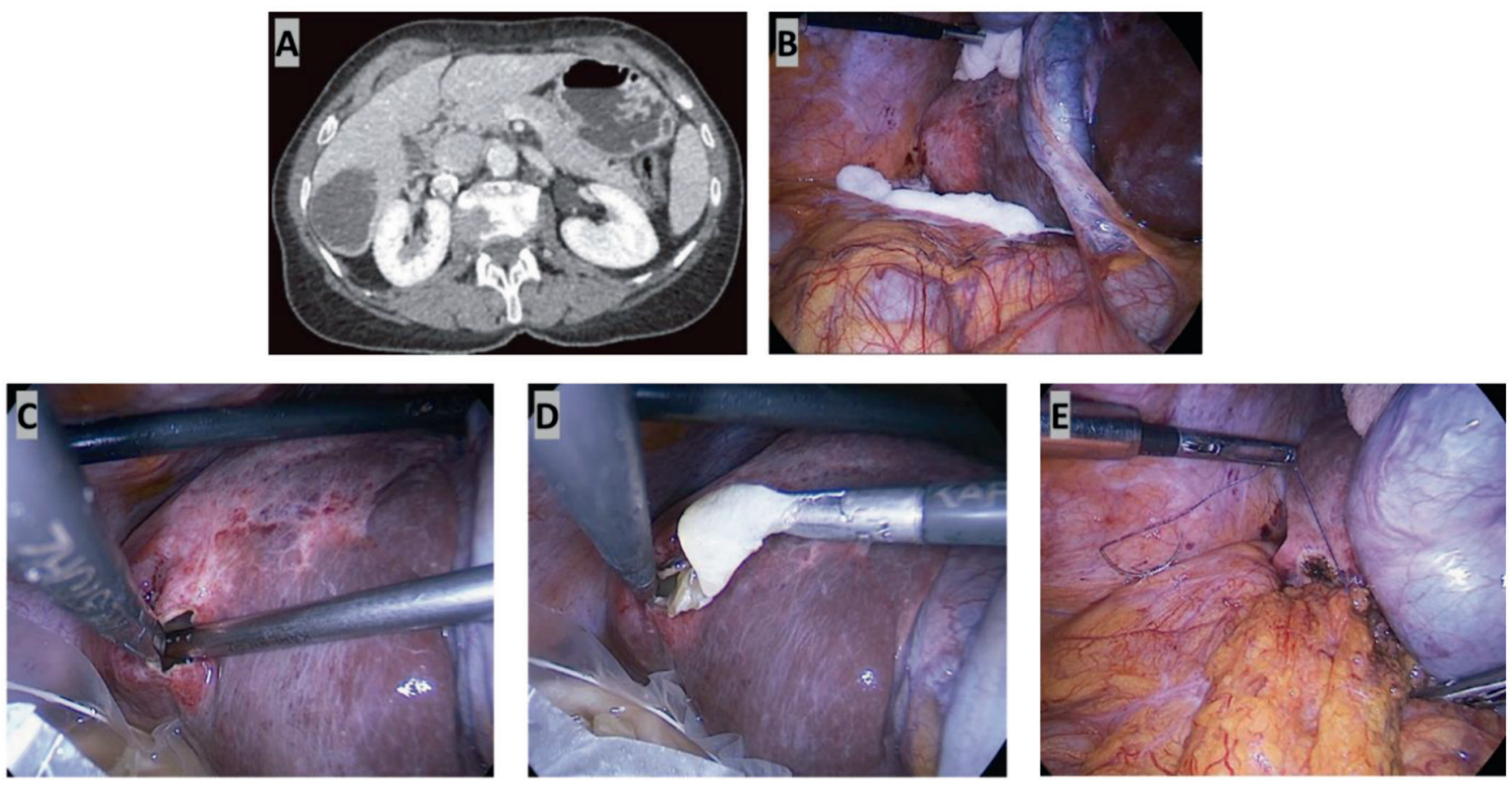

Figura 6. A: QHH, S-V1; B: LBD traccionado, QHH protegido con compresas con $\mathrm{H}_{2} \mathrm{O}_{2}$; C: Aspiración del contenido quístico; D: Extracción de vesículas hijas en bolsa; E: Omentoplastía, omento rellenando cavidad quística.

se acumulaba experiencia. La duración media de la estancia hospitalaria fue de 1 a 8 días. La mortalidad global alcanzó a 0,22\%. La morbilidad global fue de un $15 \%(0 \%-53 \%)$. La presencia de fístula biliar se encontró en un 6,24\%. La recidiva fue de un $1,09 \%$. El schock anafiláctico ocurrió en 3 pacientes $(0,3 \%)$.

\section{Recomendaciones}

- La selección adecuada de los pacientes es esencial para el éxito de la cirugía laparoscópica. El abordaje laparoscópico es seguro y técnicamente factible.

- La cirugía laparoscópica ofrece ventajas como: estancia hospitalaria más corta, menos dolor posoperatorio y una menor tasa de infección del sitio quirúrgico.

\section{Tratamiento robótico}

Los primeros 2 casos de cirugía robótica en la EQ fueron presentados en el año 2011 por Giulianotti ${ }^{23}$ y desde esa fecha se han publicado principalmente series de casos, que al día de hoy totalizan 33 pacientes ${ }^{24}$.
La cirugía hepática laparoscópica es todo un desafío técnico. Las dificultades inherentes a este procedimiento incluyen la rigidez de los instrumentos, la vista bidimensional, el temblor de la mano y la fatiga asociada con procedimientos prolongados ${ }^{25}$.

El desarrollo de un sistema robótico, teóricamente ayuda a superar algunas de estas dificultades. El robot proporciona una imagen tridimensional de alta resolución ${ }^{23,26}$, posee un diseño ergonómico, una mayor destreza y una visualización mejorada, que puede ser capaz de imitar la cirugía abierta ${ }^{27}$. Se han publicado varias series de casos, que sugieren que el uso de robótica puede tener ventajas en el control del sangrado, en la realización de hepatectomías y en la resección de quistes en segmentos difíciles (segmentos I, VII y VIII) ${ }^{28,29}$ (Figura 7).

\section{Recomendaciones}

- Las principales ventajas de esta tecnología en comparación con la cirugía laparoscópica son su diseño ergonómico, la flexibilidad de los instrumentos y una imagen amplificada.

- Sin embargo, el alto costo del equipo y su permanente mantención, impiden en la actualidad su popularización. 


\section{Tratamientos percutáneos}

Los tratamientos percutáneos se introdujeron a mediados de la década de 1980 tras la introducción de la ultrasonografía (US). Posteriormente, esta opción se ha convertido en una alternativa viable a la cirugía para determinadas etapas del quiste.

Inicialmente se desaconsejó la punción de los quistes equinococósicos debido al riesgo de schock anafiláctico causado por el eventual derrame del líquido y la posible siembra de la cavidad abdominal. Sin embargo, un número creciente de artículos ha informado de su seguridad en el tratamiento de los quistes echinococcosicos hepáticos. Un total de 4.209 quistes se han puncionado, ya sea con fines diagnósticos o terapéuticos, notificándose 16 casos de schock anafiláctico, siendo 2 de ellos mortales $(0,05 \%)^{30}$.

Estos tratamientos orientados ultrasonográficamente, tienen como objetivo destruir la capa germinal con agentes escolicidas o evacuar todo el endoquiste. El método más popular dentro del primer grupo es el PAIR (punción, aspiración, inyección de un agente escolicida y la reaspiración), mientras que varias técnicas de cateterismo pertenecen al segundo grupo. Estas últimas se basan en la aspiración del contenido sólido del quiste, la capa germinal y la laminada, a través de un catéter o dispositivo de gran calibre. Se utilizan varias variantes de estas técnicas, en particular la evacuación percutánea (PAIDS) y la técnica de cateterismo modificado (MoCaT).

$\mathrm{La}$ indicación formal del $\mathrm{PAIR}^{30}$ es para los quistes CL, CE1 y CE3a con un tamaño $<6 \mathrm{~cm}$. Se realiza mediante punción de los quistes guiada mediante US o TC con el objetivo de instalar un catéter intraquiste de calibre variable, a través del cual se aspira la mayor cantidad posible de contenido. A continuación, se instila una solución escolicida, hasta $1 / 3$ del volumen del líquido extraído, siendo las más utilizadas el etanol al $95 \%$ o la solución salina hipertónica al 30\%. Después de 10-30 min, se vuelve a aspirar el contenido quístico. Si es necesario se repite varias veces hasta obtener un líquido limpio (Figura 8).

El PAIDS $^{30}$ se utiliza para quistes $>$ de $6 \mathrm{~cm}$, luego de la punción, aspiración del contenido del quiste e inyección del agente escolicida, se inserta un catéter de 8 French en la cavidad bajo guía fluoroscópica; procediéndose a aspirar la totalidad del líquido a través del catéter y a realizar un cistograma para confirmar la no existencia de comunicaciones biliares. Se introduce alcohol absoluto al $95 \%$ en la cavidad quística para esclerosar la pared, después de 15 minutos se vuelve a aspirar todo el líquido y
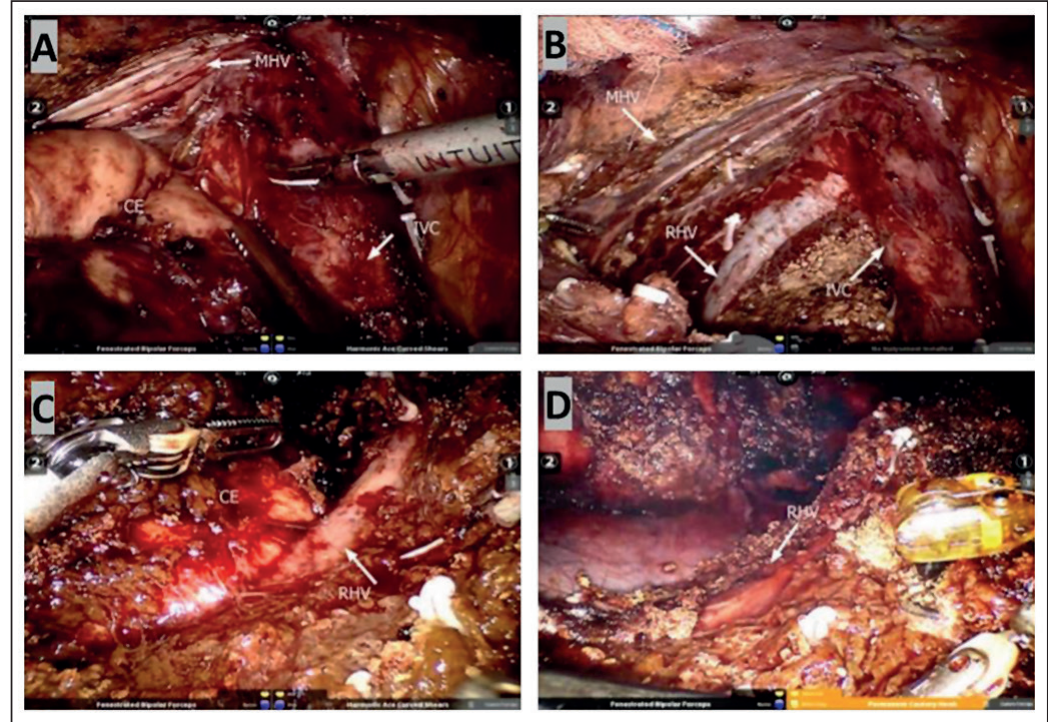

Figura 7. A: QHH, S-I; B: Resección S-I; C: QHH, S-VIII; D: Resección del S-VIII. MVH: vena hepática media; CE: QHH; IVC: vena cava inferior; RHV: vena hepática derecha. Zhao ZM et al. World Gastroenterol 2020;26:2831-38.

se retira el catéter.

El $\mathrm{MoCaT}^{30}$ se utiliza principalmente en los quistes CE2 y CE3b. Se procede a insertar un catéter 14 o $16 \mathrm{~F}$ en la cavidad del quiste, posteriormente a aspirar las membranas, quistes hijos y la totalidad del contenido y luego se irriga la cavidad con solución salina hipertónica; todo lo anterior bajo visión fluoroscópica. Después de la completa evacuación se coloca un catéter más pequeño $8 / 10 \mathrm{~F}$ que permanece hasta que el drenaje sea $<$ a $10 \mathrm{ml} /$ día.

Todos los pacientes son monitoreados durante 3-6 $\mathrm{h}$ después del procedimiento y los pacientes PAIR son dados de alta el mismo día. A aquellos que se le realiza PAIDS y/o MoCaT son hospitalizados y dados de alta al retirar el catéter. El promedio de días de estada es de 3,1 (2-9 días) ${ }^{30}$.

La seguridad y eficacia del tratamiento percutáneo de los quistes CE1 y CE3a ha sido bien demostrada con una tasa de éxito del $97,1 \%$ al $100 \%{ }^{31}$; sin embargo, en los CE2 y CE3b el éxito solamente alcanza al $39 \%^{32}$.

\section{Recomendaciones}

- PAIR está indicado para quistes $<6 \mathrm{~cm}$ en estadio CE, CE1 y CE3a, quistes accesibles múltiples, quistes infectados, recidiva posoperatoria y tratamiento médico fallido.

- PAIDS se puede realizar en quistes CL, CE1 y $\mathrm{CE} 3 \mathrm{a}>$ de $6 \mathrm{~cm}$. 


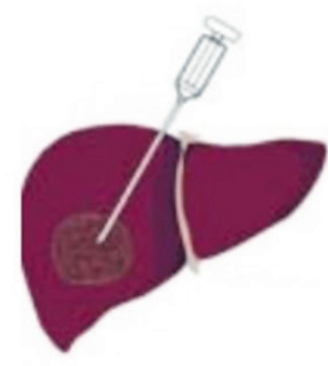

1) Punción

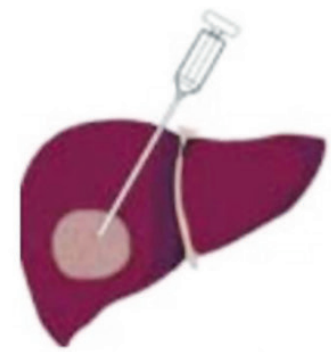

3) Inyección

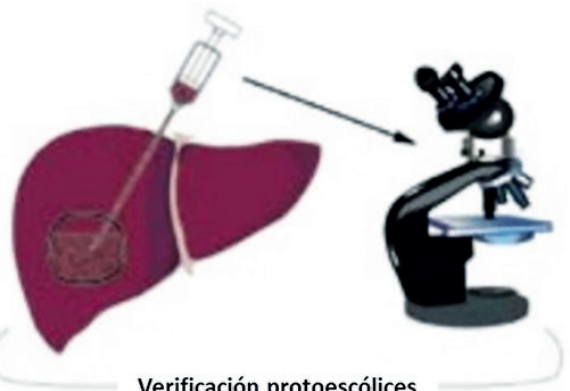

Verificación protoescólices

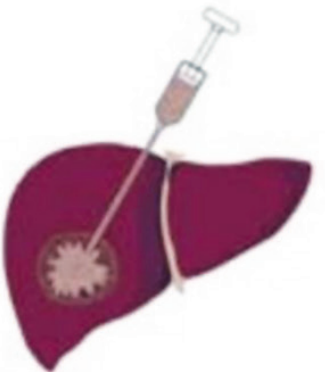

4) Reaspiración

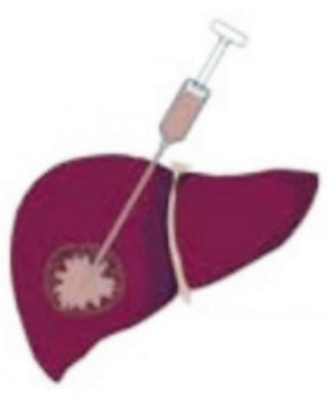

2) Aspiración

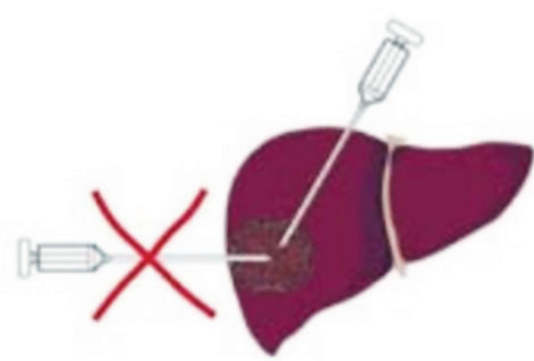

5) Ubicación punción para prevenir filtración

Figura 8. Técnica percutánea (PAIR).

- MoCaT se puede realizar en quistes CE 2 y CE3b si el paciente no es apto o rechaza cirugía.

\section{Mirar y esperar (W \& W)}

Se basa en la premisa de que la historia natural de la echinococcosis quística (EC) puede ser suficiente para el control y/o resolución de la enfermedad (13\%-25\% de los casos de EC hepática pueden resolverse espontáneamente sin tratamiento) ${ }^{33-35}$.

Los quistes en el estadio CE1 pueden degenerar espontáneamente, mientras que los estadios CE4 y CE5 considerados "inactivos" pueden ocasionalmente aumentar de tamaño y progresar, aunque generalmente tienden a permanecer estables en el tiempo. Varias declaraciones de consenso y recomendaciones de expertos en el tratamiento de la EC han respaldado el enfoque de $\mathrm{W} \& \mathrm{~W}^{4,36,37}$, lo que ha llevado a su uso cada vez mayor en pacientes asintomáticos no complicados con CE4 y CE5 hepáticos.

El estudio realizado por Luca Piccoli ${ }^{38}$, menciona que en los últimos 30 años han seguido y controlado a 690 pacientes portadores de QHH, de los cuales $127(18,4 \%)$ tenían quistes en etapa IV y V, a los cuales controlaron durante un período que fluctuó entre los 2 y 19 años, con una mediana de 52 meses; encontrando que el $97,4 \%$ de ellos continuaban inactivos.

El seguimiento de estos pacientes es importante y debe ser a largo plazo. La mayoría de las reactivaciones ocurren durante los primeros años de seguimiento, un seguimiento continuo durante al menos 3-5 años parece apropiado y prudente, aunque algunos autores recomiendan extender este período a 10 años ${ }^{37,39}$.

\section{Recomendaciones}

- Se sugiere la estrategia W\&W para el manejo de pacientes con quistes hepáticos asintomáticos y no complicados en estadios CE4 y CE5.

- Es importante conocer que el $18,4 \%$ de los pacientes, presentan quistes en etapa IV y $\mathrm{V}$ al momento del diagnóstico y, por ende, deberían ser sometidos a la estrategia de W\&W.

- El seguimiento de estos pacientes es importante y debe ser a largo plazo, de al menos 3-5 años. 


\section{Comentarios finales}

La introducción de la ecografía a finales de la década de 1970 y más tarde la tomografía computada y la resonancia magnética, revolucionaron el tratamiento clínico de la equinococosis quística beneficiando tanto a pacientes como a médicos. Las imágenes brindaron por primera vez, la posibilidad de evaluar preoperatoriamente la mejor terapia quirúrgica.

Gracias a la utilización de la ecografía en el seguimiento de los quistes hidatídicos hepáticos (QHH) asintomáticos, no complicados (CE4 y CE5), es que se ha acuñado el concepto de "observar y esperar". Esta recomendación nació de directrices dadas por la OMS-IWG, y se basa en un seguimiento a largo plazo de los quistes hidatídicos asintomáticos, demostrándose que la mayoría de los pacientes permanecen asintomáticos durante años, con un bajo riesgo de complicaciones; siendo por lo tanto innecesaria una intervención quirúrgica ${ }^{38}$.

La cirugía debe adaptarse al paciente, a los hallazgos anatómicos, a la experiencia del cirujano y a las instalaciones hospitalarias. Cabe mencionar que los QHH crecen muy lentamente y la gran mayoría de ellos son asintomáticos cuando se diagnostican. El riesgo de mortalidad de un QHH no diagnosticado debido a una eventual complicación es de un $0,5 \%$. A su vez, la incidencia de las complicaciones más temidas como la ruptura, obstrucción biliar e hipertensión portal no van más allá del $10 \%$, con una tasa de mortalidad del 5\%. Es por esto, que los procedimientos quirúrgicos realizados deben tener una mortalidad menor a estas cifras $^{39}$.

Los procedimientos mínimamente invasivos (PAIR, laparoscopía, robótica), que inicialmente estaban proscritos por la posible anafilaxia y eventual siembra, han experimentado el mismo auge y beneficios que los obtenidos en todas las otras patologías en las que se han utilizado. En la actualidad, un número creciente de artículos han informado de su efectividad y seguridad ${ }^{22}$.

En el Servicio de Cirugía del Hospital Regional de Coyhaique, en los últimos 35 años, se han intervenido 850 pacientes portadores de $\mathrm{QHH}$ con un total de 1.508 quistes. Las técnicas radicales han representado el $23,2 \%$ del total, siendo la periquistectomía total el procedimiento quirúrgico más realizado. La morbilidad general alcanzó al 17,9\%, encontrándose la fístula biliar como la complicación más frecuente con un $10,5 \%$. La mortalidad de la serie fue de un 1,4\% (presentación FLAP, XXIV Congreso Latinoamericano de Parasitología, 2016).

\section{Responsabilidades éticas}

Protección de personas y animales. Los autores declaran que para esta investigación no se han realizado experimentos en seres humanos ni en animales.

Confidencialidad de los datos. Los autores declaran que en este artículo no aparecen datos de pacientes.

Conflictos de interés: no hay.

\section{Bibliografía}

1. Jenkins DJ, Romig T, Thompson RCA. Emergence/re-emergence of Echinococcus spp. -a global update. Int J Parasitol. 2005;35:1205-19.

2. WHO.www.who.org

3. López-Bernus A, Belhassen García M, Alonso-Sardón M, Carpio-Pérez A, Velasco-Tirado V, Romero-Alegría A, et al. Surveillance of Human Echinococcosis in Castilla-Leon (Spain) between 20002012. Torgerson PR, editor. PLoS Negl Trop Dis. October 2015;9(10).

4. Brunetti E, Kern P, Vuitton DA, Writing Panel for the WHO-IWGE. Expert consensus for the diagnosis and treatment of cystic and alveolar echinococcosis in humans. Acta Trop. 2010;114:1-16.

5. International classification of ultrasound images in cystic echinococcosis for application in clinical and field epidemiological settings. Acta Trop 2003;85:253-61.

6. Stojkovic M, Rosenberger K, Kauczor HU, Junghanss T, Hosch W. Diagnosing and staging of cystic echinococcosis: ¿how do CT and MRI perform in comparison to ultrasound? PLoS Negl Trop Dis. 2012;6:e1880.

7. Mihmanli M, Idiz UO, Kaya C, Demir U, Bostanci O, Omeroglu S, et al. Current status of diagnosis and treatment of hepatic echinococcosis. World J Hepatol. 2016;8:1169-81.

8. Sözüer E, Akyuz M, Akbulut S. Open surgery for hepatic hydatid disease. Int Surg. 2014;99:764-9.

9. Smego RAJr, Sebanego P. Treatment options for hepatic cystic echinococcosis. Int J Infect Dis. 2005;9:69-76.

10. Yuksel O, Akyurek N, Sahin T, Salman B, Azili C, Bostanci H. Efficacy of radical surgery in preventing early local recurrence and cavity-relate complications in hydatic liver disease. J Gastrointest Surg. 2008;12:483-9.

11. Pascal G, Azoulay D, Belghiti J, Laurent A. Hydatid disease of the liver. In: Jarnagin WR, editor. Blumgard's Surgery of the Liver Biliary tract, and Pancreas. 6th edn. Philadelphia: Elsevier; 2017 p. 1102-21.

12. Jerraya H, Khalfallah M, Osman SB, et 
al. Predictive factors of recurrence after surgical treatment for liver hydatid cyst. Surg Endosc. 2015;29:86-93.

13. Berberoglu M, Taner S, Dilek ON, Demir A, Sari S. Gasless vs gaseous laparoscopy in the treatment of hepatic hydatid disease. Surg Endosc. 1999;13:1195-8.

14. Goya S, Saha SK, Yadav SK, Tiwari A, Soinet SA. Surgical approaches to hepatic hydatidosis ranging from partial cystectomy to liver transplantation. Ann Hepatobiliary Pancreat Surg. 2018; 22:208-15.

15. Ertem M, Karahasanoglu T, Yavuz N, Erguney S. Laparoscopically treated liver hydatid cysts. Arch Surg. 2002;137:11703.

16. Baltar J, Baamonde I, Concheiro P, García L, Brenlla J, Escudero B, et al. Tratamiento laparoscópico de los quistes hidatídicos hepáticos: técnicas y complicaciones postoperatorias. Cir Esp. 2009;86:33-7.

17. Nguyen KT, Camblin TC, Geller DA. World review of laparoscopic liver resection-2804 patients. Ann Surg. 2009;250:831-41.

18. Buell JF, Cherqui D, Geller DA, O'Rourke N, Iannitti D, Dagher I, et al. The international position on laparoscopic liver surgery: the Louisville Statement 2008. Ann Surg. 2009;250:825-30.

19. Kathkouda N, Fabiani P, Bnizri E, J Mouiel. Laser resection of a liver hydatid cyst under video laparoscopy. Br J Surg. 1992;79:560-1.

20. Pinto P, Vallejos C, Cruces E, Lobos J, Hernández F, Ríos M y col. Tratamiento laparoscópico del quiste hidatídico hepático. Rev Chil Cir. 2011;63:361-7.

21. Jarufe N, Galindo JL, Bachler JP, Ahumada V, Rebolledo R, Crovari F, et al. Radical Laparoscopic Treatment for Hepatic Hydatid Disease. J Gastrointest Dig Syst. 2016;6:419.
22. Tuxun T, Zhang JH, Zhao JM, Tai QW, Abudurexti M, Ma HZ, et al. World review of laparoscopic treatment of liver cystic echinococcosis-914 patients. Int J Infect Dis. 2014;24:43-50.

23. Giulianotti PC, Bianco FM, Daskalaki D, González-Ciccarelli LF, Kim J, Benedetti E. Robotic liver surgery: technical aspects and review of the literature. Hepatobiliary Surg Nutr. 2016;5:311-21.

24. Magistri P, Pecchi A, Franceschini E, Pesi B, Guadagni S, Catellani B, et al. Not just minor resections: robotic approach for cystic echinococcosis of the liver. Infection 2019;47:973-9.

25. Ban D, Kudo A, Ito H, Mitsunori Y, Matsumura S, Aihara A, et al. The difficulty of laparoscopic liver resection. Updates Surg. 2015;67:123-8.

26. Lai ECH, Tang CN. Training robotic hepatectomy: the Hong Kong experience and perspective. Hepatobiliary Surg Nutr. 2017;6:222-9.

27. Ho CM, Wakabayashi G, Nitta H, Ito N, Hasegawa Y, Takahara T. Systematic review of robotic liver resection. Surg Endosc. 2013;27:732-9.

28. Abu Hilal M, Aldrighetti L, Dagher I, Edwin B, Troisi RI, Alikhanov R, et al. The Southampton Consensus Guidelines for Laparoscopic Liver Surgery: From Indication to Implementation. Ann Surg. 2018; 268: 11-18.

29. Araki K, Kubo N, Watanabe A, Kuwano H, Shirabe K. Systematic review of the feasibility and future of laparoscopic liver resection for difficult lesions. Surg Today 2018;48:659-66.

30. Guven Kahriman, Nevzat Ozcan, Serap Dogan, Oguz Karaborklu. Percutaneous treatment of liver hydatid cysts in 190 patients: a retrospective study. Acta Radiol 2017;58:676-84

31. Koroglu M, Erol B, Gurses C, Türkbey B, Baş CY, Alparslan AS, et al. Hepatic cystic echinococcosis: percutaneous treatment as an outpatient procedure. Asian Pac J Trop Med 2014;7:212-5.

32. Kabaalioglu A, Ceken K, Alimoglu E, Apaydin A. Percutaneous imaging-guided treatment of hydatid liver Kahriman et al. 683 cysts: ¿do long-term results make it a first choice? Eur J Radiol. 2006; 59:65-73.

33. Keshmiri M, Baharvahdat H, Fattahi SH, Davachi B, Dabiri RH, Baradaran H, et al. Albendazole versus placebo in treatment of echinococcosis. Trans R Soc Trop Med Hyg. 2001;95:190-4.

34. Romig T, Zeyhle E, Macpherson CN, Rees PH, Were JB. Cyst growth and spontaneous cure in hydatid disease. The Lancet 1986;1(8485):861.

35. Li T, Ito A, Pengcuo R, Sako Y, Chen X, Qiu D, et al. Post-treatment follow-up study of abdominal cystic echinococcosis in tibetan communities of northwest Sichuan Province, China. Garcia HH, editor. PLoS Negl Trop Dis. 2011;5:e1364.

36. Larrieu E, Frider B, del Carpio M, Salvitti JC, Mercapide C, Pereyra R, et al. Asymptomatic carriers of hydatidosis: epidemiology, diagnosis, and treatment. Rev Panam Salud Pública 2000;8:250-6.

37. Menezes da Silva A. Hydatid cyst of the liver-criteria for the selection of appropriate treatment. Acta Trop. 2003;85:237-42.

38. Piccoli L, Tamarozzi F, Cattaneo F, Mariconti M, Filice C, Bruno A, et al. Long-term sonographic and serological follow-up of inactive echinococcal cysts of the liver: hints for a «watch-andwait» approach. PLoS Negl Trop Dis 2014;8:e3057.

39. Kern P, Menezes da Silva A, Akhan O, Müllhaupt B, Vizcaychipi KA, Budke C, et al. The Echinococcoses: Diagnosis, Clinical Management and Burden of Disease. Adv Parasitol. 2017;96:259-369. 